\title{
ETUDE ET EXTRACTION DES PROPRIETES DE SURFACE PAR TELEDETECTION SPATIALE DANS LES COURTES LONGUEURS D'ONDE
}

\author{
Bernard Pinty* and Michel M. Verstraete** \\ *LERTS, 18 Avenue Edouard Belin, 31055 Toulouse Cedex, France \\ ${ }^{* *}$ AOSS, The University of Michigan, Ann Arbor, MI 48109-2143, U.S.A.
}

\section{RESUME}

Les satellites constituent a priori un excellent moyen expérimental pour caractériser et assurer le suivi, à des échelles de temps et d'espace adéquates, des propriétés des surfaces continentales. Ils peuvent donc apporter beaucoup à l'analyse des phénomines climatiques mettant en cause les perturbations de ces propriétés de surface. Que ce soit pour valider des résultats de modeles climatiques, pour spécifier leurs conditions initiales a la limite inférieure, ou pour extraire des informations physiques quantitatives de données de télédétection spatiale, des modeles physiques représentant le transfert de rayonnement i la surface sont nócessaires. Un modèle analytique nouveau exprimant la réflectance bidirectionnelle sur des surfaces est présenté. Les surfaces sont considértes comme des milieux poreux constitués d'éléments optiquement homogènes. Une attention particulière est accordbé au traitement des effets dus à l'orientation des éléments et à leur arrangement relatif dans le milieu. Ce modłle a été inversé et validé a partir de mesures effectuées au laboratoire, sur le terrain et par avion. Ce modele offre de nouvelles perspectives quant a l'analyse et l'interprét ation de données satellitaires, et permet de spécifier des conditions limites sur les continents dans les modèles de climat.

\section{INTRODUCTION}

La menace d'une modification du climat de la terre potentiellement causée par les activités humaines a motivé la mise en place par les organiomes internationaux du Programme International Géosphère Biosphère (PIGB). Parmi les axes majeurs déinis par le PIGB, les études du comportement des écosystèmes terrestres occupent une place de choix aussi bien du point de vue de leurs modifications possibles et de leurs adaptations à des changements climatiques, que du point de vue de leurs effets de rétroaction sur ces mêmes changements climatiques. Les enjeux sont évidemment d'une importance majeure. Ils débordent largement le cadre strictement écologique et concernent, par exemple, aussi bien les activités économiques dans les secteurs industriels et agricoles a court et moyen termes, que, aplus long terme, les modes de vie des habitants de nombreuses régions sensibles du globe.

Les changements climatiques identifiables font intervenir plusieurs aspects, avec des effets de rétroaction dans la biosph̀̀re plus ou moins marqués. Parmi ces aspects, le plus connu est certainement l'effet de serre directement causé par l'augmentation en concentration de gas tels que le gas carbonique $\left(\mathrm{CO}_{2}\right)$, le methane $\left(\mathrm{CH}_{4}\right)$, et les chlorofluorocarbones (CFCa) dans l'atmosphère. Cette augmentation résulte à la fois des injections directes à partir de diverses sources liées aux activités humaines, et de la deatruction de la biomasse active par des opérations de déforestation. Cette dégradation de la couverture végétale entraíne deux effets : d'une part une diminution de la capacité de fixation du gas carbonique atmoophérique et d'autre part une augmentation importante des émissions dans l'atmosphère de $\mathrm{CO}_{2}, \mathrm{CH}_{4}, \mathrm{~N}_{2} \mathrm{O}$ et d'autres gas. En plus de cette contribution directe de la déforestation à l'effet de serre, cette opération modifie également de manière drantique et immédinte l'état des surface concernés à travers l'albédo, la rugosité, le ruissellement et d'autres parametres physiques qui conditionnent les bilans d'énergie et d'eau à la surface. Cet exemple de la déforestation illustre le fait que les changements d'origine anthropogénique ou naturelle de l'état des surfaces terrestres ont donc globalement deux types d'impact 'sur le climat en modifiant les flux biogéophysiques et les flux biogéochimiques. La chaîne des interactions possibles entre climat et état de surface est extrêmement longue et difficile à décrire, notamment parce qu'elle fait intervenir, à des échelkes de temps et d'espace qui sont très variables, des phénomènes dont les mécanismes sont insuffisamment connus et documentes.

Etant donné le niveau de complexité des interactions entre les différentes composantes du système clinatique (l'atmosphère, la biosphère, ls terre solide, les océans et la cryosphère), les modèles numériques sont indispensables pour analyser et prédire les changements de climat résultant d'un forçage interne. La qualité des résultats fournis par les modèles est bien evidemment dépendante du niveau de compréhension que l'on a de l'ensemble des processus intervenants et de la qualité de leur représentation sous forme paramétrée aux échellesd éfinies par la maille des modèles, mais également et surtout de la diaponibilité en calculateurs suffisamment puissants pour effectuer le volume de calcul nécessaire /1/ (la prochaine génération de calculateurs doit notamment permettre d'augmenter très sensiblement la résolution des modèles). Dans l'état actuel des choses et avec toutes les imperfections et les limitations qui sont reconnues, la plupart des modèles de climat indique une augmentation potentielle marquée de la température de la terre (entre $0.05^{\circ} \mathrm{C}$ et $0.6^{\circ} \mathrm{C}$ par décade) en simulant des scénarios d'émisaions continues en gas à effet de serre $/ 2 /$. Les principales incertitudes qui existent sur la validite de ces simulations proviennent de traitements encore préliminaires et insatisfaisants de l'effet de rétroaction des nuages, de la réponse des régiona glactes aux hautes latitudes, du cycle hydrologique en surface et du couplage des océans avec les autres composantes du système climatique. Les grandes différences qui apparaissent dans ces résultats de simulation concernent les impacts a échelle régionale (au sens de grandes régions climatiques duglob e) lorsque ces simulations sont effectuées avec des modzles tridimensionels permettant de spatialiser les informations $/ 3 /$. En effet, selon le modèle considéré et pour une région donnfe, les résultats peuvent indiquer aussi bien une augmentation de plusieurs degrés en température avec une diminution des précipitations que l'inverse, ou tout autre combinaison de variations de ces deux paramètres météorologiques qui sont 
des variables clé pour ce qui concerne la répartition géographique et l'évolution des grands écooystèmes terrestres.

La qualité des simulations du syatème climatique et leurs améliorations potentielles passent notamment par un contrôle ou une validation des résultats a partir de bases de donnéesd' observation. Du point de vue expérimental, les données satellitaires apparaissent a priori comme les seules capables d'apporter des élémenta de solutions adaptés aux échelles concerntes. L'intérêt majeur de ces données est de permettre l'estimation de quantités moyennés sur un domaine d'espace et avec une résolution temporelle adequate, tont en assurant une bonne cohérence dans la precision des mesures et une couverture d l'echelle du globe. Mis a part les problèmes techniques tels que la calibration ou la dérive des capteurs, la contrepartie de ces avantages eat l'interprétation physique de ces mesures qui est souvent délicate et pas immédiatement en rapport avec des variables importantes pour le bilan d'eau et d'énergie a la surface. Ainai, les mesures effectuées par satellite correspondent a des mesures radiatives et, seuls les paramètres physiques primaires du milieu qui conditionnent explicitement la luminance dans les courtes longueurs d'onde ou l'émission dans le domaine thermique et micro-onde peuvent être raisonnablement restitués par l'inversion de modèles physiques avec ces mesures. Dane une seconde phase, ces paramètres physiques sont potentiellement utilisables pour inverser des modeles de processus permettant d'estimer un second ensemble de paramètres d'un intérêt plus immédiat pour la modélisation du climat ou l'analyse de situations particulières. Du point de vue de la dialectique entre données de télédétection spatiale et modéles climatiques, on peut raisonnablement considérer que les modeles sont indispeneables pour définir les variables importantes à estimer ainsi que la précision nécenasire à atteindre aux échelles d'espace et de temps qui sont judicieux. Dans le contexte actuel, il semble que les efforts doivent tout d'abord porter sur les variables de surface qui contrôlent les flux biogéophysiques.

Pour lea mêmes raisons et avec les mêmes limitations que celles mentionnées plus haut, les donnés satellitaires constituent a priori un excellent outil pour suivre l'b́volution des fcosystemes terrestres et leurs éventuelles perturbations par des causes naturelles ou anthropogéniques. L'identification et le suivi des phénomènes de déforestation et de désertification, ainsi que la destruction de la biomasse active par les feux sont autant d'exemples simples qui illustrent les apports des données satellitaires dans la problematique du PIGB. Des travaux récents montrent de manière tentative que les possibilités d'estimations quantitatives de variables sophistiquees, comme la production primaire /4/ et le flux de $\mathrm{CO}_{2} / 5 /$, existent. La plupart de ces travaux reposent sur des analyses empiriques du Normalized Difference Vegetation Index (NDVI) qui correspond à une combinaison du canal visible et proche infrarouge du capteur AVHRR embarqué sur les satellites de la série NOAA. Les données issues de ce capteur vont prendre une importance grandissante pour analyser l'état et l'évolution des surfaces car elles constituent un compromis acceptable du point de vue des bandes spectrales utilisés, de la taille des pixels, et surtout de la pérennité des mesures. Ainsi, au moment où les donnés des futures plateformes EOS seront disponibles, on devrait disposer d'une vingtaine d'années de données AVHRR, ce qui constitue une banque d'informations à partir de laquelle des modifications sensibles des grands éconystèmes terrestres pourraient être identifiables. Cependant, la encore, ce projet ne peut être mené a bien que si l'on est capable d'interpréter le contenu physique des mesures obtenues par télédétection apatiale de maniłre a suivre l'évolution des parametres primaires et a savoir s'affranchir de perturbations comme celles dues aux effets atmosphériques ou celles essentiellement causées par des variations des conditions de géométrie d'observation.

Le propos de cet article est de résumer un développement théorique permettant d'interpréter les données de reflectances mesurtes dans le domaine des courtes longueurs d'onde. L'approche choisie est conditionnée par la nécessité de satisfaire aux spécifications techniques des modèles climatiques (comme par exemple un faible coût de calcul), et de développer un modłle qui puisse être facilement inversé avec des données de télédétection. Cette stratégie vise donc à assurer un pontage entre diverses communautés scientifiques, en définissant un outil commun aux modélisateurs du climat, aux téledétecteurs et aux Écologistes.

\section{MODELE DE REFLECTANCE BIDIRECTIONNELLE}

En temps que premiłre étape vers une interprét ation quantitative généralisé des mesures de réflectance bidirectionnelle, nous avona développé un modele applicable aux cas de surfaces homog̀̀nes et semi-infinies du point de vue de leurs propriétés optiques. Ce type de surface eat considéré comme un milieu poreux dont la réflectance dépend des propriétés optiques des éléments composant la surface (c'est-à-dire des grains pour un sol nu ou des feuilles pour les couverts végétaux), mais également de l'orientation moyenne de ces éléments et de leur positionnement relatif dans le milieu. Dans ces conditions, Verstraete et al. /6/ et Pinty et al. /7/ ont montré que la réflectance bidirectionnelle s'écrit analytiquement de la manière suivante:

$$
\rho\left(\theta_{1}, \phi_{1} ; \theta_{2}, \phi_{2}\right)=\frac{\omega}{4} \frac{\kappa_{1}}{\kappa_{1} \mu_{2}+\kappa_{2} \mu_{1}}\left[\tilde{P}_{v}(G) P(g)+H\left(\mu_{1} / \kappa_{1}\right) H\left(\mu_{2} / \kappa_{2}\right)-1\right]
$$

où

$$
\begin{aligned}
\mu_{1} & =\cos \theta_{1} \\
\mu_{2} & =\cos \theta_{2} \\
\cos g & =\cos \theta_{1} \cos \theta_{2}+\sin \theta_{1} \sin \theta_{2} \cos \left(\phi_{1}-\phi_{2}\right) \\
H(x) & =\frac{1+x}{1+\sqrt{(1-\omega)} x} \\
G & =\left[\tan ^{2} \theta_{1}+\tan ^{2} \theta_{2}-2 \tan \theta_{1} \tan \theta_{2} \cos \left(\phi_{1}-\phi_{2}\right)\right]^{1 / 2}
\end{aligned}
$$

Dans ces équations, $\theta_{1}$ et $\phi_{1}$ désignent le sénith et l'asimuth du soleil, $\theta_{2}$ et $\phi_{2}$ désignent le sénith et l'asimuth du capteur, $g$ eat l'angle de phase entre le rayon incident et le rayon réfléchi, $\omega$ est l'albédo de diffusion simple des éléments constituant la surface, $P(g)$ est la fonetion de phase, $\kappa_{1}$ et $\kappa_{2}$ décrivent la distribution en orientation des éléments de la surface, et le terme $H\left(\mu_{1} / \kappa_{1}\right) H\left(\mu_{2} / \kappa_{2}\right)-1$ approxime la contribution de la diffusion multiple au sein du milieu, d'après Dickinson et al. $/ 8$.

$\mathcal{P}_{v}(g)$ est la fonction qui exprime la transmission conjointe du rayonnement incident et réfléchi, et de fait décrit l'effet de 'hotspot' qui est particulitrement marqué aux faibles angles de phase. Cette fonction peut être approximée par la relation suivante:

$$
\begin{aligned}
& \bar{P}_{v}(G) \approx 1+\frac{1}{1+V_{p}(G)} \\
& V_{p}(G)=4\left(1-\frac{4}{3 \pi}\right) \frac{G}{2 r \Lambda} \frac{\mu_{2}}{\kappa_{2}}
\end{aligned}
$$


où $r$ désigne le rayon typique des tâches illuminées sur les éléments inclinés de la surface et $\Lambda$ est la densité de ces éléments dans le milieu.

En pratique, pour estimer $\rho\left(\theta_{1}, \phi_{1} ; \theta_{2}, \phi_{2}\right)$, Pinty et al. /7/ ont suggéré d'utiliser la fonction de HenyeyGreenstein et une paramétrisation proposée par Goudriaan pour exprimer respectivement les fonctions $P(g)$ et $\kappa$. Dans sa forme finale, seulement quatre parametres sont nécessaires pour calculer la réflectance bidirectionnelle d'une surface: $\omega$ et $\theta$ qui décrivent les propriétés optiques intrinséques des éléments par l'albédo de diffusion simple et le facteur d'asymétrie de leur fonction de phase, un paramètre noté $\chi_{\ell}$ par Goudriaan /9/ qui indique l'orientation moyenne des lémemts $\left(\chi_{\ell}=0\right.$ pour une distribution uniforme, $\chi_{\ell}=0.6$ pour un milieu planophile et $\chi_{\ell}=-0.4$ pour un milieu érectophile), et enfin le paramètre noté $2 r \Lambda$ qui intervient dans la fonction $V_{p}(g)$ et qui décrit la distance moyenne typique entre les éléments. On peut, si cela est nécessaire, augmenter ce nombre minimum de quatre paramètres pour représenter des effets supplément aires comme par exemple la composante speculaire de la fonction de phase. Dans le cas d'un milieu optiquement dense et dano la limite de la diffusion primaire, Dickinson et al. /8/ ont évalué analytiquement l'albédo de surface à partir de l'équation (1). Cette solution généralise la formulation proposée par Sellers /10/ qui ne a'applique que pour les cas d'une diffusion homogène.

Le modele présenté ci-dessus pouvant être appliqué indifféremment dans les longueurs d'onde visibles et proche infrarouges du spectre, il est facile de proposer une formulation analytique du NDVI pour un couvert optiquement homogène. Pour des raisons de clarté, on peut considérer ici que les eléments de la surface sont isotropes, c'est-d-dire que le flux transmis est égal au fux réfléchi, et que le milieu est suffisamment dense et observé loin des conditions de 'hot spot', c'eat-a-dire $\tilde{P}_{v}(g) \approx 1$. En négligeant la contribution de la diffusion multiple du canal visible, ce qui est raisonable pour des couverts végétaux, Pinty et al. /11/ ont établi la relation suivante:

$$
N D V I \approx \frac{\omega_{2} H_{2}\left(\mu_{1} / \kappa_{1}\right) H_{2}\left(\mu_{2} / \kappa_{2}\right)-\omega_{1}}{w_{2} H_{2}\left(\mu_{1} / \kappa_{1}\right) H_{2}\left(\mu_{2} / \kappa_{2}\right)+w_{1}}
$$

où les indices 1 et 2 font respectivement référence aux canaux visible et proche infrarouge du capteur AVHRR. Cette relation montre qu'en dépit des simplications effectuées sur le cas de surfaces simples, des effets directionnels sont encore présents sur le NDVI. Ces effets proviennent de la contribution en diffusion multiple du canal infrarouge qui ne peut être négligée sur on couvert végétal en raison des grandes valeurs prises par le paramètre $\omega$ dans cette région spectrale. Compte tenu des biais syatématiques sur les angles d'éclairement et d'observation des écosystèmes terrestres en fonction de la latitude, cette dépendance angulaire du NDVI peut perturber l'analyse des variations à échelle globale de cet indice.

EXTRACTION DES PARAMETRES DE SURFACES

Comme cela est indiqué en introduction, la description et le suivi temporel de l'état des surfaces continentales nécessitent la mise au point de procédures permettant d'inverser les mesures de réflectance bidirectionnelle pour en extraire les paramètres physiques primaires du milieu.

Une telle procédure a été propośée et testée par Pinty et al. /12/ sur des surfaces de sols nus, puis appliquée a des couverts végétaux $/ 7,8 /$. Cette procédure repose sur l'utilisation d'un algorithme itératif permettant de déterminer simultanément les valeurs optimales des quatre parametres primaires intervenants dans l'équation (1). Les difficultes essentielles proviennent de la non-linéarité de l'équation (1), et les tests de sensibilité effectués par /12,13/ sur un algorithme d'optimisation non-linéaire montrent les limitations et les performances de ce type de procédure. Ces tests de sensibilité concernent la dépendance et la stablité des résultats vis-a-vis (i) des valeurs initiales données au schéma, (ii) des erreurs aléatoires sur les données de reffectance, (iii) de la relation fonctionnelle entre les paramètres a estimer et la réflectance bidirectionnelle. Une attention particulìre doit être accord́́e aux problèmes relatifs a l'obtention d'une solution unique et a la répartition des erreurs d'entrées sur les valeurs des paramètres physiques restitués par inversion, puisque, les incertitudes expérimentales étant quantifiables, ceci conditionne la précision avec laquelle la valeur des paramètres de surface peut être estimé. L'ensemble de ces points de techniques numériques sont d'une importance fondamentale pour apprécier l'apport potentiel des données satellitaires a l'estimation quantitative de paramètres caractérisant les propristes radiatives des écosystèmes continentaux.

Pinty et al. / 7 / ont montré que l'ensemble du schéma (modèle + inversion) fourni des résultats de bonne qualité et donc que les problemes évoqués ci-dessus peuvent être adéquatement pris en compte. Ainsi, la valeur du résidu de la procédure d'inversion est compris entre 5 et $10 \%$ des valeurs des réftectances, ce qui est comparable aux valeurs des incertitudes sur les observations. De plus, a partir de mesures effectuées sur du soja, Pinty et al. /7/ ont validé les résultate du schéma d'inversion en comparant les valeurs des coefficients de transmission, absorption et réflection des feuilles déduites des résultats de l'inversion (ces coefficients sont calculables explicitement en connaissant les parametres $\omega$ et $\theta$ ), avec celles obtenues indépendamment par des mesures de laboratoire. Ces résultats sont très prometteurs, mais ils demandent a être confirmés par des expériences plus completes. Dans un premier temps, il est raisonnable de faire porter les efforts sur la validation du schéma développé pour les surfaces optiquement homogènes et semi-infinies, avant d'aborder le cas plus réaliste de surfaces complexes constituées par un milieu présentant des hétérogénéités optiques. CONCLUSIONS

L'interprétation phyoique des données satellitaires acquises dans le domaine des courtes longueurs d'onde est un des éléments clés qui doivent permettre de décrire quantitativement l'état passé, actuel et futur des tcosystèmes continentaux. La stratégie a developper pour extraire les informations utiles du signal doit être élaborte en un minimum de trois phases qui sont (i) la décontamination du signal des effets atmosphériques, (ii) l'inversion des donnés avec des modèles physiques pour restituer la valeur des parametres physiques conditionnant le transfert radiatif a la surface, et (iii) l'estimation de paramètres d'environnement par inversion de modeles de processus utilisant, comme données d'entrée, les valeurs des paramétres radiatifs déterminés précédemment.

Le travail présenté dans cet article s'inè̀re dans la deuxième phase de cette stratégie, il fournit des résultats encourageants pour ce qui concerne le traitement de surfaces optiquement simples. Une étude est en coura de développement pour aborder le ca de surfaces mixtes composétes d'un maximum de deux milieux possédant des propriétés optiques différentes. Les résultate préliminaires laissent entrevoir que, par rapport au cas précédent, ce problème diffre dans la difficulté par au moins un ordre de grandeur. Il est donc nécessaire de bien asseoir les bases d'une démarche d'ensemble en travaillant do manière plus approfondie et diversifíe sur des surfaces simples. Bien que la démarche apparaisse lourde, voire fastidieuse, c'eat le prix a payer pour une utilisation rationnelle de données de télédétection spatiale dans le cadre du PIGB. 


\section{REMERCIEMENTS}

Une partie du travail présenté ici a été réalioé au National Center for Atmospheric Research (NCAR) en collaboration avec $R$. E. Dickinson. B. Pinty remercie le Centre National d'Etudes Spatiale (CNES) pour son soutien. Ce travail a ette financé en partie par le Programme National de Télédétection Spatiale (PNTS). M. Verstraete remercie le Department of Atmonpheric, Oceanic and Space Sciences de l'Université du Michigan, ainsi que le Eros Data Center du USGS pour leur oupport financier à ce travail.

\section{REFERENCES}

/1/Verstraete, M. M., Land Surface Processes in Climate Models: Status and Prospects, in Climate and the Geoseiences : A Challenge for Science and Society in the 21 ${ }^{\text {tt }}$ Century, Edité par A. Berger, S. Schneider, et J. C. Duplessy, Kluwer Academic Publimers, 321-340 (1989).

/2/Jaeger, J., Developing Policies for Responding to Climatic Change, World Climate Program Impact Studies, WMO/TD $\mathrm{N}^{\circ} 225$ (1988).

/3/Kellogg, W. and Z-C. Zhao, Sensitivity of Soil Moisture to Doubling of Carbon Dioxide in Climate Model Experiments. Part I : North America Journal of Climate, 4, 348-366 (1988).

/4/Tucker, C. J. and P. J. Sellers, Satellite Remote Sensing of Primary Production, International Journal of Remote Sensing, $7,1395-1416$ (1986).

/5/Tucker, C. J., I. Y. Fung, C. D. Keeling, and R. H. Gammon, Relationship between Atmospheric $\mathrm{CO}_{2}$ Variations and a Satellite-derived Vegetation Index, Nature, 819, 195-199 (1986).

/6/Veratraete, M. M., B. Pinty, and R. E. Dickineon, A Physical Model of the Bidirectional Reflectance of Vegetation Canopies; Part 1: Theory, Journal of Geophysical Research, 95, 11755-11766 (1990).

/7/Pinty, B., M. M. Veratraete, and R. E. Dickinson, A Physical Model of the Bidirectional Reflectance over a Vegetative Canopies; Part 2: Inversion and Validation, Journal of Geophysical Research, 05, 11767-11775 (1990).

/8/Dickinson, R. E., B. Pinty, and M. M. Verstraete, A Theoretical and Practical Basis for Relating Surface Albedos in GCMs to Remotely Sensed Data, Agricultural and Forest Meteorology, 52 (1990).

/9/Goudriaan, J., Crop Micrometeorology: A Simulation Study, Wageningen Center for Agricultural Publishing and Documentation, Wageningen, The Netherlands, (1977) p. 249.

/10/Sellers, P. J., Canopy Reflectance, Photosynthesis and Transpiration, International Journal of Remote Sensing, 6, 13351372 (1985).

/11/Pinty, B., M. M. Verstraete, and R. E. Dickinson, A Physically-based Model for the NDVI, in: $4^{\text {th }}$ AVHRR Date Usere' Meeting, 105-109, Eumetsat, Rothenburg, F.R.Germany (1990).

/12/Pinty, B., M. M. Verstraete, and R. E. Dickinson, A Physical Model for Predicting Bidirectional Reflectance over Bare Soil, Remote Sersing of Environment, 2T, 273-288 (1989).

13/Pinty, B. and M. M. Verstraete, Extracting Information from Bidirectional Reflectances, Journal of Geophysical Researeh, in press (1990). 